\title{
Incorporation of excluded-volume correlations into Poisson-Boltzmann theory
}

\author{
Dmytro Antypov, ${ }^{1, *}$ Marcia C. Barbosa, ${ }^{2, \dagger}$ and Christian Holm ${ }^{3,1, \$}$ \\ ${ }^{1}$ Max-Planck-Institut für Polymerforschung, Ackermannweg 10, 55128 Mainz, Germany \\ ${ }^{2}$ Instituto de Física, UFRGS, 91501-970, Porto Alegre, RS, Brazil \\ ${ }^{3}$ Frankfurt Institute for Advanced Studies (FIAS), Johann Wolfgang Goethe Universität, Max-von-Laue-Strße 1, D-60438 \\ Frankfurt am Main, Germany
}

(Received 15 February 2005; published 22 June 2005)

\begin{abstract}
We investigate the effect of excluded-volume interactions on the electrolyte distribution around a charged macroion. First, we introduce a criterion for determining when hard-core effects should be taken into account beyond standard mean-field Poisson-Boltzmann (PB) theory. Next, we demonstrate that several commonly proposed local-density-functional approaches for excluded-volume interactions cannot be used for this purpose. Instead, we employ a nonlocal excess free energy by using a simple constant-weight approach. We compare the ion distribution and osmotic pressure predicted by this theory with Monte Carlo simulations. They agree very well for weakly developed correlations and give the correct layering effect for stronger ones. In all investigated cases our simple weighted-density theory yields more realistic results than the standard PB approach, whereas all local density theories do not improve on the PB density profiles, but on the contrary, deviate even more from the simulation results.
\end{abstract}

DOI: 10.1103/PhysRevE.71.061106

PACS number(s): 61.20.Qg, 82.70.Dd, 87.10.+e

\section{INTRODUCTION}

Understanding the behavior of charged macroions in solution is an important problem in fundamental science [1] as well as in industrial [2] and biological applications [3]. Charged stabilized colloidal dispersions are present in paints, inks, and pharmaceutical products and are used in the fabrication of nanostructured materials [4-6]. These systems serve also as a primitive model for the crowded cellular environment that represents numerous biomacromolecules and cellular polymers $[7,8]$. What all the applications above have in common is that when a charged macroion is immersed in an electrolyte solution, it is surrounded by counterions to balance the surface charge. The charged macroion surface along with the neutralizing diffuse layer of counterions is usually referred to as the electric double layer, the understanding of which is crucial for describing the behavior of such systems. For instance, the stability of colloidal dispersion depends on the distribution of small ions around the colloid. The electrophoretic mobility of the solution also can be rationalized in terms of the ion distribution [9-12] and most of the electrochemical reactions occur in this interfacial region [13].

As a result, there has been a considerable effort to describe the density profile around the macroion for different macroion geometries. The earliest theory that had significant success was the Poisson-Boltzmann (PB) approach. Its versions for planar geometry, the so-called Gouy-Chapman theory $[14,15]$, can be solved exactly. It also has an analytical solution for an infinitely long linear macroion confined to a cylindrical cell $[16,17]$, whereas only a numerical solution

\footnotetext{
*Electronic address: antypov@mpip-mainz.mpg.de

†Electronic address: barbosa@if.ufrgs.br

"Electronic address: c.holm@fias.uni-frankfurt.de
}

can be obtained in the case of a spherical geometry. The major flaw of this mean-field approach is that it neglects all correlations between the ions. For a long time, integralequation theories have been developed to adequately describe dense systems of electrolytes, and recently field theories have become very popular in calculating correlation corrections to the mean field PB approach; see, e.g., Refs. $[3,18,19]$ for overviews. However, since the treatment of size effects is mixed with the electrostatic correlations, in many approaches it becomes difficult to identify the role of each effect. And finally integral equation theories work well at high densities when excluded-volume contributions are very strong, whereas they are problematic in the low-density regime.

It would therefore be desirable to have a theoretical framework which retains the simplicity of the early attempts, but also accommodates correlation effects-something that can be done within density functional theories. It is possible to rigorously rewrite the partition function of, say, a system of charged colloids, as a density functional [20], in which the contribution beyond the mean field is included as an additive correlation correction to the free-energy density. The functional form of this correction is unknown and one has to use a reasonable ansatz for it. The spirit is very similar to the fundamental problem of integral equations, where one also has to make an educated guess (namely, the closure relation). However, in the case of a functional this involves a freeenergy density expression rather than a relation between twoand three-point functions. It thus relies on a different kind of intuition and thus permits some complementary insight.

A number of density-functional prescriptions for taking both size and electrostatic correlations into account have been proposed [21-24]. These theories are able to reproduce to some extent the density profile of charged systems. However, since they treat both size and electrostatic correlations together, the origin of the result is not clear. Recently we adopted a different approach. We studied systems of point- 
like counterions (therefore no size effects) and addressed the question of when the electrostatic correlations become relevant. For treating these correlations we proposed the Debye-Hückel hole-cavity functional $[25,26]$. This theory relies on a Debye-Hückel treatment of the one-component plasma (OCP) [27-29], in which the short-distance failure of linearization is overcome by postulating a correlation hole. Since beyond a certain density the resulting OCP free-energy density is a concave function of density, this favors the development of inhomogeneities. In the pure OCP these are balanced by the homogeneously charged background. However, if one uses the OCP free-energy density as a correlation correction to the mean-field functional describing the double layer at a charged surface, one has all the charge opposite to the counterions located on that surface, rather than homogeneously distributed as a stabilizing background. The consequence is that the double layer becomes unstable and all ions collapse onto the surface, an effect which has been termed "structural catastrophe" $[30,31]$. To prevent this effect we introduced a spherical exclusion region where no background can be found. The prescription for finding the size of such an exclusion serves both to keep the theory selfconsistent and to establish the range of validity of the PB approach. Comparisons of the ionic charge distribution around a charged cylinder and a charged sphere showed a very good agreement with the simulations for both monovalent and trivalent counterions [26].

Having studied how to take into account the electrostatic correlations, we address in this paper the relevance of excluded-volume correlations. We present a validity criterion for the PB approach by constructing a parameter whose value approximately indicates when excluded-volume correlations are expected to become relevant. We then test several local density approaches that have been advocated $[32,33]$, demonstrating that they all fail to take the size correlations into account and that they even lead to an instability of the solution beyond a certain ion size. In order to circumvent this, a weighted density functional based on a simplified Tarazona approach [34-36] is introduced. Our results are compared with Monte Carlo (MC) simulations, showing very good agreement for the cases of moderately developed hardcore correlations and even for strongly electrostatically interacting systems in both zero salt and nonzero salt cases.

The remainder of the paper is organized as follows. In Sec. II we discuss validity of the PB approach for a colloidal system with non-point-like counterions and show how the size effect can be incorporated into the model. Details of the used numerical methods are given in Sec. III. These are followed by the results and discussions presented in Sec. IV, and our conclusion in Sec. V.

\section{SIZE CORRELATIONS WITHIN DENSITY- FUNCTIONAL THEORY}

Consider a spherical colloid of radius $r_{c}$ and negative charge $Z$, which is located in the center of a spherical cell of radius $R_{c}$. This cell represents a bulk colloidal solution with colloid volume fraction $\phi=\left(r_{c} / R_{c}\right)^{3}$. The counterions are taken as positively charged hard spheres of diameter $a$ and

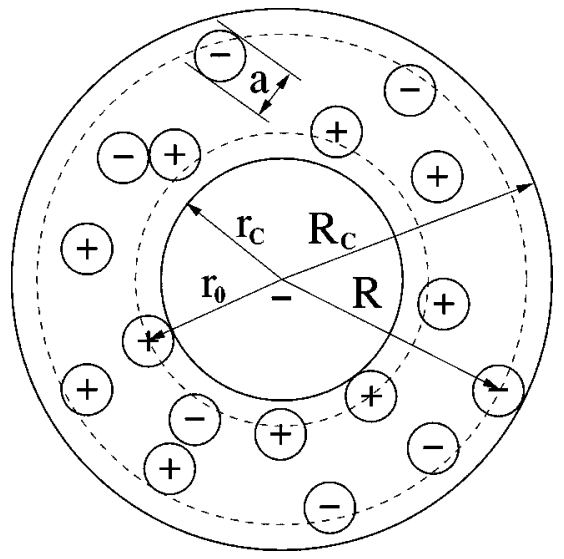

FIG. 1. Colloidal cell model. The system is defined by five independent parameters: number of counterions, $N$, number of salt ions, $N_{s}$, and three characteristic sizes $r_{0}, R$, and $a$ measured in units of $l_{B} v^{2}$. See the Appendix for more details.

valence $v$, and $N=Z / v$ of them provide the neutrality of the cell. The solvent is modeled as a uniform dielectric background of dielectric constant $\epsilon$, and the strength of the electrostatic interactions is defined by Bjerrum length

$$
l_{B}=\frac{q^{2}}{4 \pi \epsilon_{0} \epsilon k_{B} T},
$$

where $q$ is the unit charge. In the nonzero salt case, $N_{s}$ positive and $N_{s}$ negative salt ions are also included. Here we assume that all ions have the same size and valence as the counterions. The average charge distribution is described by local densities $n_{-}(r)$ for the coions and $n_{+}(r)$ for the counterions and positive salt ions. These are defined for $r_{0} \leqslant r \leqslant R$, where $r_{0}=r_{c}+a / 2$ is the distance of closest approach between the macroion and particles and $R=R_{c}-a / 2$ (see Fig. $1)$. Therefore, $\phi_{e}$, the volume fraction of the electrolyte in the cell confined between $r_{c}^{3}$ and $R_{c}$ is given by

$$
\phi_{e}=\frac{a^{3}\left(N+2 N_{s}\right)}{8\left(R_{c}^{3}-r_{c}^{3}\right)} .
$$

The effective surface charge density $\sigma$ should be defined in terms of $r_{0}$ rather than $r_{c}$-i.e., $\sigma=Z /\left(4 \pi r_{0}^{2}\right)$. This will be used later when defining the plasma parameter of the system.

The central task of a density-functional theory is to derive an analytical expression for the free-Helmholtz-energy functional that upon minimization gives the density profiles of the free ions in solution. Its simplest form is given by the Poisson-Boltzmann functional: namely

$$
\begin{aligned}
F_{P B}= & \int d^{3} r\left(k_{B} T n_{+}(\mathbf{r})\left\{\ln \left[n_{+}(\mathbf{r}) \lambda^{3}\right]-1\right\}+k_{B} T n_{-}(\mathbf{r})\right. \\
& \left.\times\left\{\ln \left[n_{-}(\mathbf{r}) \lambda^{3}\right]-1\right\}+f_{e l}(\mathbf{r})\right),
\end{aligned}
$$

which includes the translational entropy of the ions $(\lambda$ is the thermal de Broglie wavelength) and all electrostatic interactions represented by $f_{e l}(\mathbf{r})$. The electrostatic interactions within the mean-field approximation are given by 


$$
f_{e l}(\mathbf{r})=v q\left[n_{+}(\mathbf{r})-n_{-}(\mathbf{r})\right] \psi(\mathbf{r}),
$$

where $\psi(\mathbf{r})$ is the total electrostatic potential created at position $(\mathbf{r})$ by the fixed macroion and all ions together. The minimization of Eq. (3) with respect to $n_{+}(\mathbf{r})$ and $n_{-}(\mathbf{r})$ gives the Boltzmann density distributions

$$
n_{ \pm}(\mathbf{r})=n_{ \pm}^{0} e^{\mp \beta v q \psi(\mathbf{r})},
$$

where parameters $n_{+}^{0}$ and $n_{-}^{0}$ are defined by the charge neutrality condition. In spherical geometry, Eq. (5) together with the Poisson equation

$$
\nabla^{2} \psi(r)=-\frac{4 q v \pi}{\epsilon}\left[n_{+}(r)-n_{-}(r)\right]
$$

and the boundary conditions at $r=r_{0}$ and $r=R$ comprises a fully defined Poisson-Boltzmann problem. The problem with this approach is that the ions are considered as point charges in some average electric field and both electrostatic and excluded-volume correlations between them are not taken into account. When do these correlations matter? Comparing the PB predictions to simulation results, one has found out that, for pointlike ions, electrostatic correlations become relevant when the plasma parameter $\Gamma_{2 d}=\sqrt{\pi \sigma l_{B}^{2} v^{3}}$ becomes larger than $1[25,26,37-40]$. Here we address the question under what condition the excluded-volume effects become significant. For a uniform hard-sphere liquid we know that the radial distribution function changes from monotonically decaying to nonmonotonic at volume fractions of the order of $\phi \approx 0.2$, which will be the reference volume fraction in our further estimates. In the case of confined liquids, ions tend to concentrate close to the surface and their concentration at the surface, especially in charged systems, may be much higher than that in the bulk [41]. Therefore, to access the hard-core effects one should consider the average volume fraction within the first layer of counterions close to the colloid surface. Since the colloid is much larger than the ions, it can be approximated as (for simplicity the salt is not included)

$$
\phi_{s}=\frac{\pi a^{2}}{6} \int_{0}^{a} n_{P B}(x) d x,
$$

where $n_{P B}(x) \ell^{3}=\Gamma_{2 d}^{4} / \pi\left(2 \bar{x} \Gamma_{2 d}^{2}+1\right)^{2}$ is the exact solution obtained in the planar geometry $[14,15,42]$. Here $\bar{x}=x / \ell$, where $\ell=l_{B} v^{2}$. After integration we find that the volume fraction close to the macroion is given by

$$
\phi_{s}=\frac{\hat{a}^{3} \Gamma_{2 d}^{4}}{3\left(2 \Gamma_{2 d}^{2} \hat{a}+1\right)},
$$

where $\hat{a} \equiv a / \ell$. Then, for $\phi_{s} \lesssim 0.2$, there are only weakly developed excluded-volume correlations, and the PB approximation should be still valid. For larger values of $\phi_{s}$ we expect to see some layering effects close to surfaces. This criterion is strictly valid only for a planar geometry, but is expected to approximately hold for sufficiently large spherical or cylindrical macroions where the curvature effects $(\propto 1 / R)$ are negligible. An analogous formula which takes the curvature into account can also be derived for a cylindrical
PB cell for which the contact density is known analytically. Since electrostatic correlations were not taken into account, we do not expect this simple analysis to hold for $\Gamma_{2 d} \geqslant 2$. Beyond this value, the force-distance curves between charged plates cease to be monotonic, and beyond $\Gamma_{2 d}$ $\simeq 2.45$ attractions even between like charged macroions can occur [37]. These effects are the results of correlations between different double layers (like, for instance, ion interlocking $[38,43])$ that are stronger than the size effects we are describing here. Note that Eq. (8) is designed to give a limit of validity of the mean-field approach. For high ionic radius $\phi_{s}$ can become larger than 1 , losing its relation to the actual volume fraction in the system.

Correlations can be included in the PB model by adding to the PB free energy $F_{P B}$ an excess free-energy term,

$$
F=F_{P B}+F_{e x} .
$$

The excess free energy $F_{e x}$ originates from internal interactions within the system, and it is unknown. In principle, it can account for both the hard-core repulsion and the electrostatic correlations. Since we want to test the excludedvolume effects within the range of ionic strength in which they overcome the electrostatic correlations, the latter will be neglected within our approach.

There are a number of functionals which can be used to include hard-core effects in uniform liquids of a given density $n$. Within the local density approximation (LDA) one can choose one of these free-energy density expressions, $f_{e x}[n]$, and replace the uniform density by a local one so that the total excess free energy reads

$$
F_{e x}=k_{B} T \int d^{3} r n(r) f_{e x}[n(r)] .
$$

For our system we can take $n(r)=n_{+}(r)+n_{-}(r)$ which means that the hard-core effects are treated identically for both positive and negative ions. The idea behind Eq. (10) is quite simple. A particle at $\mathbf{r}$ is supposed to be affected by only the particles around it, in a range given by the interaction. If the range of the interparticle interaction is much smaller than the typical length for variations in $n(r)$, the system can be divided into small subvolumes of nearly constant density and each of them can be treated as part of a homogeneous system. If we take ions as charged hard spheres, we can use the free-energy density derived, for example, from the Carnahan-Starling (CS) equation of state [44]: namely

$$
f_{C S}[\phi(r)]=\frac{\phi(r)[4-3 \phi(r)]}{[1-\phi(r)]^{2}},
$$

where $\phi(r)=\pi a^{3} n(r) / 6$ is the volume fraction occupied by the free ions. For denser liquids, the accuracy might be improved by using the more precise virial expansion for the Percus-Yevick theory for hard spheres [45]: namely,

$$
\begin{aligned}
f_{\text {vir }}[\phi(r)]= & {\left[4 \phi(r)+5 \phi(r)^{2}+6.12 \phi(r)^{3}+7.02 \phi(r)^{4}\right.} \\
& \left.+7.905 \phi(r)^{5}+9.4208 \phi(r)^{6}\right] .
\end{aligned}
$$

The last two expressions agree up to second order in the local volume fraction $\phi(r)$. 
A simple form of $F_{e x}$ can also be derived from the freevolume $(f v)$ expression for a lattice gas [32,33]: namely,

$$
\left.F_{f v 1}=k_{B} T \int d^{3} r\left[\frac{1}{a^{3}}-n(r)\right] \ln \left[1-n(r) a^{3}\right)\right],
$$

where $a$ is the lattice spacing. With this form of functional the excluded-volume effects can be explicitly incorporated into the PB equation. However, its density expansion is different from that of a hard sphere. Another expression based on the free-volume concept which can be found in Ref. [46],

$$
F_{f v 2}=-k_{B} T \int d^{3} r n(r) \ln \left(1-\frac{\phi(r)}{2}\right),
$$

gives lower-order density terms similar to Eqs. (11) and (12).

The assumption of smooth variations of $n(r)$ being within the characteristic range of hard-core interactions is only valid for sufficiently small ionic diameters $a$. Consequently, as we will show later, all the functionals above underestimate the densities close to the colloid and completely fail to give the correct density profile in the more interesting cases where stronger variations in $n(r)$ are observed. Moreover, all expressions above have a singularity at a certain value of volume fraction. This reflects the fact that the bulk density cannot exceed some upper limit. If a local density is higher than that-for example, due to a charged surface - the local density functional does not converge and no density profile can be obtained.

In order to circumvent the failure of the local density approach, a number of weighted-density approaches (WDA's) have been proposed [34-36,47-50]. Initially the new methods were developed for the description of neutral hard-sphere solutions. Stimulated by the success of the WDA for neutral systems, some rather complex and involved methods have already been proposed for charged suspensions $[21,22,51]$. The prescription we have followed here is in the spirit of the generalized van der Waals theory of Nordholm and co-workers [50]. We represent the nonlocality of the free-energy density functional through a coarse-grained density distribution $\bar{n}(r)$. The weighted density is a nonlocal functional of the local density $n(r)$. This can be pictured as a mean density around point $\mathbf{r}$ averaged over a volume related to the range of the interactions. In this context, the local density in Eq. (10) is replaced by some weighted density $\bar{n}(r)$ : namely,

$$
F_{e x}=k_{B} T \int d^{3} r n(r) f_{e x}[\bar{n}(r)],
$$

where

$$
\bar{n}(r)=\int d^{3} r^{\prime} w\left(\left|\mathbf{r}-\mathbf{r}^{\prime}\right|\right) n\left(\left|\mathbf{r}^{\prime}\right|\right) .
$$

The connection between the real system and the approximated functional comes from the weight function $w\left(\left|\mathbf{r}-\mathbf{r}^{\prime}\right|\right)$, which should be chosen to give reasonable direct correlation functions which are functional derivatives of $F_{e x}[\bar{n}]$. The most important are the first- and second-order correlation functions, defined as

$$
\begin{gathered}
c^{(1)}(r)=\frac{\delta F_{e x}[\bar{n}]}{\delta n(r)}, \\
c^{(2)}\left(r, r^{\prime}\right)=\frac{\delta^{2} F_{e x}[\bar{n}]}{\delta n(r) \delta n\left(r^{\prime}\right)} .
\end{gathered}
$$

In the approach of Tarazona et al. $[35,36,47]$ one assumes that the weight itself is also density dependent and can be expanded in powers of the weighted density as follows:

$$
w\left(\left|\mathbf{r}-\mathbf{r}^{\prime}\right|\right)=w_{0}(r)+w_{1}(r) \bar{n}(r)+w_{2}(r) \bar{n}(r)^{2}+\cdots .
$$

If we substitute this expression into the direct correlation function in Eq. (17), the resulting expansion can be set equal to the direct correlation function of a uniform hard-sphere fluid [52]. This way it is possible to obtain the weight function that up to second order is given by $[35,36,47]$

$$
\begin{aligned}
& w_{0}(r)=\frac{3}{4 \pi a^{3}} \Theta[a-r], \\
& w_{1}(r)=\left[0.475-0.648 \frac{r}{a}+0.113\left(\frac{r}{a}\right)^{2}\right] \quad(r<a) \\
& =\left[0.288 \frac{a}{r}-0.924+0.764 \frac{r}{a}-0.187\left(\frac{r}{a}\right)^{2}\right] \\
& (a<r<2 a) \\
& =0 \quad(r>2 a), \\
& w_{2}(r)=\frac{5 \pi a^{3}}{144}\left[6-12 \frac{r}{a}+5\left(\frac{r}{a}\right)^{2}\right] \quad(r<a) \\
& =0 \quad(r>a) .
\end{aligned}
$$

Since our aim is not to precisely describe the hard-sphere effects but just to access their relevance, we will employ the simplest form of the weight function that is the first term in Eq. (18) or a constant weight [50] given by Eq. (19). We will refer to this weight as WDA0 whereas the weight defined by Eqs. (19)-(21) will be called WDA2 and will be used to validate our results. For a pure hard-sphere fluid, WDA0 reproduces the discontinuity in the direct correlation function predicted by Percus and Yevick $[46,53]$. However, it overestimates the range of the correlation function, especially at high densities, when compared to the density-dependent weights $[21,34]$ or to direction-dependent weights $[22,49,51]$. Having this in mind, we will concentrate on the systems for which size plays a relevant role but the differences between the constant weight and more sophisticated approaches do not affect our main conclusions.

Measuring all lengths in units of $\ell=l_{B} v^{2}$ reveals that the full partition function of our cell model depends on five system parameters. The observables-for example, the reduced density profile $\hat{n}(r)=n(r) \ell^{3}$-remain constant under rescaling which does not change the following quantities: the number of counterions, $N=Z / v$, the number of salt ions, $N_{s}$, the 
reduced distance of closest approach, $\hat{r}_{0}=r_{0} / \ell$, the $r_{0} / R$ ratio, and the reduced ion diameter $\hat{a}=a / \ell$ (see the Appendix). The same holds for PB theory and is also true for our WDA theory. For the WDA it implies that both the WDA freeenergy correction and the weight function have to obey this restriction.

\section{NUMERICAL METHODS}

In this section we give details of the numerical methods used to study the cell system described in Sec. II. Three different ways were employed to find the ion distribution in the cell. The first one was a direct Monte Carlo simulation of the cell model which gave us reference data to test the theoretical results. The density profile minimizing a given freeenergy functional was obtained using numerical iteration until it converged to the equilibrium charge distribution. Another way of minimizing the functional was by Monte Carlo sampling. Some technical details of these three methods are summarized below.

\section{A. Monte Carlo simulation}

Within this approach we simulate the cell model exactly as it is-all ions are taken as charged hard spheres of diameter $a$ confined between two spherical shells of radii $r_{c}$ and $R_{c}$. To gather the statistics of charge distribution the ions are moved around the cell and a single-ion move is either accepted or rejected according to the usual Metropolis probability:

$$
\pi=\min [1, \exp (-\beta \Delta E)],
$$

where $\Delta E$ is the difference between the system internal energy after and before the move. Since the density profile is highly anisotropic, a combination of two types of moves was found to improve the efficiency of the sampling. An ion was either inserted at a random position in the cell or randomly displaced within a cube centered at its current position. The former allowed for efficient exploration of low-density regions, whereas the latter proved to be efficient close to the colloid where a successful insertion of an ion could be a rare event due to the high packing fraction. The frequency of using one or the other move as well as the displacement range were adjusted to give an about $50 \%$ acceptance rate.

\section{B. Iterative functional minimization}

When minimizing a functional containing a nonzero correlation term, Eq. (5) becomes dependent on the excess chemical potential

$$
\mu_{ \pm}^{e x}(r)=\frac{\delta F_{e x}[n(r)]}{\delta n_{ \pm}(r)}
$$

and reads

$$
n_{ \pm}(r)=n_{ \pm}^{0} e^{\mp \beta v q \psi(r)-\beta \mu_{ \pm}^{e x}(r)} .
$$

Once the expressions for $\mu_{ \pm}^{e x}$ are derived for a given functional, we can find the ion distribution which satisfies both Poisson equation and Eq. (24). Integrating the Poisson equa- tion over a spherical shell of radius $r$ and using the Gauss theorem, an integro-differential equation for the electric field $E(r)$ can be obtained. Consequently, the optimum density profile can be obtained from the numerical iteration of this equation until convergence is achieved.

\section{Monte Carlo functional minimization}

Within this approach the ion position is described only by its distance from the colloid, $r$, and this uniquely defines the density distribution $n(r)$. Each MC step consists of moving an ion to a new trial position $r_{0}<r<R$. This move is either accepted or rejected with probability [54]

$$
\pi=\min [1, \exp (-\beta \Delta F)],
$$

where $\Delta F$ is the free energy difference after and before the move and it is explicitly given by the functional we minimize. This method was found to be more stable and worked much faster than the iterative procedure, though the final result did not depend on the numerical approach used.

\section{RESULTS AND DISCUSSION}

In this section we compare how well the different densityfunctional approaches described in Sec. II capture the excluded-volume interactions. First, we apply numerical techniques described in Sec. III to two colloidal systems already studied in the literature [33]. Then we perform a systematic analysis of hard-core effects by investigating a number of different systems with and without added salt.

We start from considering two salt-free systems which were also used in [33] to study the excluded-volume effect in colloidal solutions. In both systems $r_{0}=50 \AA, R=100 \AA, a$ $=10 \AA$, and $l_{b}=7 \AA$. The number of monovalent ions is different, and it is $N=200$ in system (a) and $N=500$ in system (b). Note that already for 200 ions some packing effects are expected to be seen because $\phi_{s}=0.25$. Figure 2 shows the ion density distribution close to the colloid obtained using different approaches for both (a) and (b). Comparison between the $\mathrm{PB}$ and the MC curves for system (a) reveals that hard-core repulsion decreases condensation by pushing ions away from the colloid. This effect is captured well by the WDA, whereas it is significantly overestimated by all LDA's - the predicted contact densities are too low. The highest packing fraction achieved in LDA calculations for system (a) was below the critical value and the equations converged.

In the case of 500 ions, the LDA with functional given by Eq. (13) was found to be numerically unstable. The iterative functional minimization failed to converge, while some convergence could be still achieved by explicitly limiting the highest density at $a^{-3}$ in the MC sampling of the functional. The result, however, depended on the number of bins and therefore was not physical. A plateau close to the colloid, as seen in [33], was observed only for relatively large bin sizes, while smaller bins resulted in a sawlike density profile (not shown here). The other LDA's, while still converging, overestimated hard-core effects and also resulted in unphysical profiles. Moreover, all local approaches missed the layering clearly captured by WDA0 and observed in simulations at 

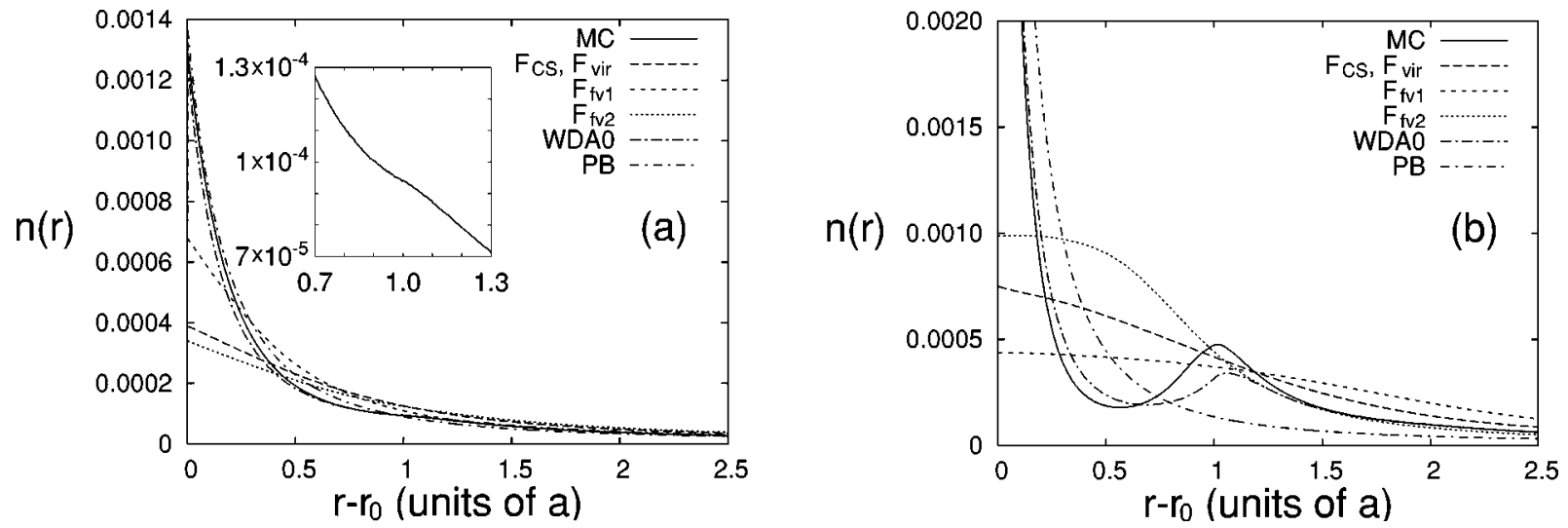

FIG. 2. Ion distribution close to the colloid surface measured in systems with $r_{0}=50 \AA, R=100 \AA, a=10 \AA$, and $l_{B}=7 \AA$ and containing (a) $N=200$ and (b) $N=500$ monovalent ions (units of $n(r)$ are $\AA^{-3}$ ). Each curve corresponds to a particular method used: MC is the result of the Monte Carlo simulation; $F_{C S}, F_{v i r}, F_{f v 1}$, and $F_{f v 2}$ are obtained using the LDA with a form of the excess free energy given by Eqs. (11)-(14), correspondingly; WDA0 is the constant-weight curve and PB is the numerical solution of PB equation without any hard-core corrections.

distance $a$ from the colloidal surface [Fig. 2(b)].

Below we consider a model system in which parameters $r_{0}$ and $R=5 r_{0}$ are kept fixed, whereas the ion size and the Bjerrum length are varied. The cell containing $N=100$ monovalent ions of size ranging between $0.1 r_{0} \leqslant a \leqslant 0.8 r_{0}$ is studied at four Bjerrum lengths $0.1 r_{0}, 0.2 r_{0}, 0.3 r_{0}$, and $0.4 r_{0}$. These correspond to the plasma parameter $0.5 \leqslant \Gamma_{2 d} \leqslant 2.0$ which enables us to investigate hard-core effects in systems with weak, moderate, and strong electrostatic correlations. The parameter $\phi_{s}$ calculated for each of these 32 systems and given in Table I predicts that, at all Bjerrum lengths, the size correlations are expected to be seen for ion sizes $a \geqslant 0.3 r_{0}$, where $\phi_{s}$ is already greater than 0.2 .

The Monte Carlo data show that, indeed, the ion density profile is concave for $a=0.1 r_{0}$ and $a=0.2 r_{0}$ but develops a convex region at a distance about $a$ from the colloid surface for larger ion sizes at all four Bjerrum lengths. This indicates some packing taking place which is well captured by our $\phi_{s}$ criterion. Figure 3 shows both the reference Monte Carlo and WDA0 density profiles calculated for different ion sizes at fixed Bjerrum length $l_{B}=0.2 r_{0}\left(\Gamma_{2 d}=1.0\right)$. The development of layering with increasing the ion size is well captured by the nonlocal-functional approach, whereas none of the LDA's exhibits any layering and therefore are not shown in Fig. 3.

Another way of checking how well correlations are captured by a particular excess free-energy functional is to com- pute the osmotic pressure. In real systems this pressure also depends on correlations between ions of different cells, something which is not taken into account within the cell model approximation. So by pressure we refer to the pressure exerted on the rigid wall at $r=R$ of our cell model. Within the simulations, the pressure is given [42] by the contact density at $r=R$ :

$$
\Pi=k_{B} \operatorname{Tn}(R) .
$$

For the density-functional approach, this exact expression should be corrected [55] to recover the free-energy functional after integrating pressure over the volume. The correction term is generally small, and for simplicity we will directly compare contact densities predicted by different methods. Figure 4 shows both the colloid contact density $n\left(r_{0}\right)$ and boundary density $n(R)$ given by the Monte Carlo simulations and different local and nonlocal density approaches for systems at fixed Bjerrum length $l_{B}=0.1 r_{0}\left(\Gamma_{2 d}\right.$ $=0.5$ ) as a function of the ionic diameter $a$. The colloid contact density is informative of how well a certain method works at the most packed region of the system and can also be related to the pressure. Figure 4(b) shows that the local approaches underestimate $n\left(r_{0}\right)$ at high ionic radius when compared to the simulations. We found that even for small ionic sizes the PB density profile was closer to the MC reference data than the results of any of the locally "improved"

TABLE I. Parameter $\phi_{s}$ of different ionic sizes (columns), Bjerrum (rows) lengths, and plasma parameter (rows). Both Bjerrum lengths $l_{B}$ and ion sizes $a$ are given in units of $r_{0}$. Packing effects are expected to be seen in those systems for which $\phi_{s}>0.2$. Unrealistically high values of $\phi_{s}$ observed for large ions indicate inapplicability of PB theory and failure of the LDA for these systems.

\begin{tabular}{ccccccccc}
\hline \hline$l_{B} / r_{0}\left(\Gamma_{2 d}\right) \downarrow a / r_{0} \rightarrow$ & 0.1 & 0.2 & 0.3 & 0.4 & 0.5 & 0.6 & 0.7 & 0.8 \\
\hline $0.1(0.5)$ & 0.01 & 0.08 & 0.23 & 0.44 & 0.74 & 1.13 & 1.59 & 2.13 \\
$0.2(1.0)$ & 0.02 & 0.11 & 0.28 & 0.53 & 0.87 & 1.29 & 1.79 & 2.37 \\
$0.3(1.5)$ & 0.03 & 0.13 & 0.31 & 0.57 & 0.92 & 1.35 & 1.86 & 2.46 \\
$0.4(2.0)$ & 0.03 & 0.13 & 0.32 & 0.59 & 0.95 & 1.38 & 1.91 & 2.51 \\
\hline \hline
\end{tabular}



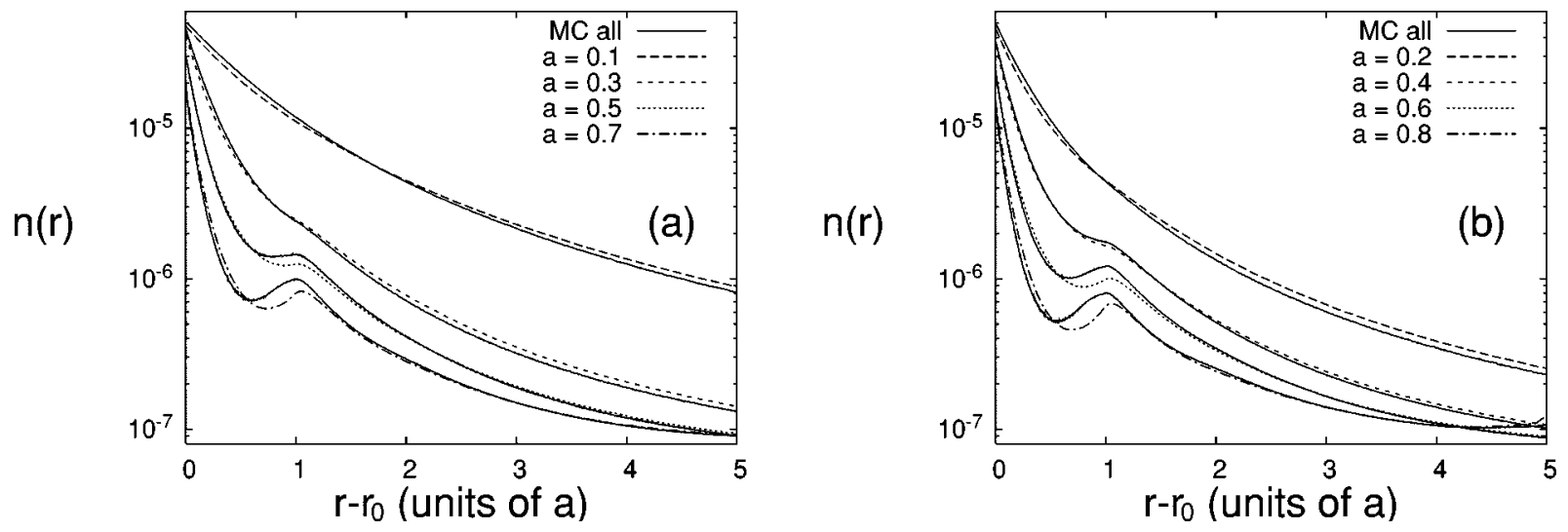

FIG. 3. Ion distribution close to the colloid surface measured in systems with plasma parameter $\Gamma_{2 d}=1.0$. Odd (a) and even (b) ionic diameters are shown separately for sake of clarity. Only WDA0 curves are marked while all MC curves are presented as solid lines and can be identified by the corresponding closest dashed WDA0 curve. All distances are measured in ionic diameters, so the formation of the second layer of counterions is always expected to be around 1 . The units of number density are $r_{0}^{-3}$.

functionals. Moreover, the limitation of the local approach is illustrated by the failure of the LDA's to converge at large $a$ (no data are shown for large ions). Both WDA's we used here give consistent results when compared to MC simulations.

Now, we concentrate on the case of relatively large ions of diameters $a \geqslant 0.4 r_{0}$ for which layering is clearly observed. According to the $\phi_{s}$ criterion, the density profiles of such systems should deviate from PB theory and the hard-core interactions can be even more significant than the electrostatics in some cases. Figure 5 shows the integrated ion fraction

$$
P(r)=\frac{1}{N} \int_{r_{0}}^{r} d r 4 \pi r^{2} n(r)
$$

for systems with ionic diameters fixed at (a) $a=0.4 r_{0}$ and (b) $a=0.6 r_{0}$ for plasma parameter $\Gamma_{2 d}=0.5,1.0$, and 2.0. Clearly, a larger plasma parameter leads to an increased condensation (the curves are shifted up) - an effect which is governed by the electrostatics and also present in PB theory. One could expect that for high $\Gamma_{2 d}$ electrostatic correlations would be a

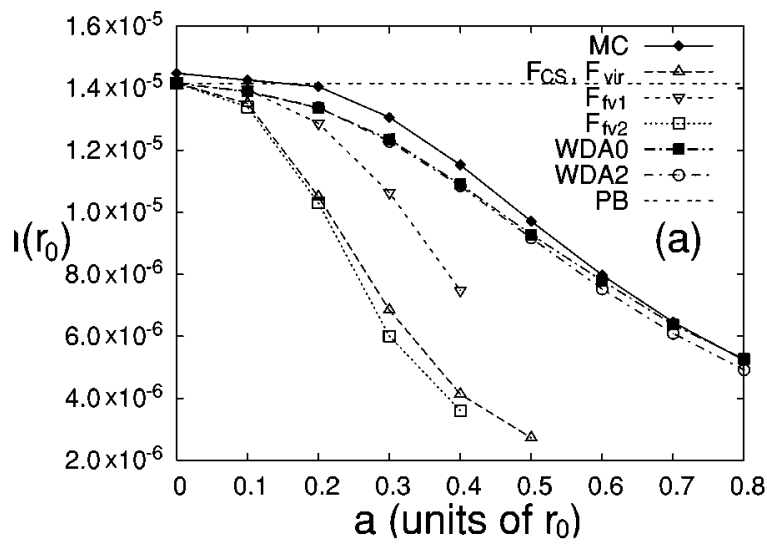

dominant effect [26], and significant deviations to PB theory and also to our WDA corrected DFT should arise due to electrostatic correlations, which we did not account for. However, under the investigated circumstances, the ionic size plays a more relevant role. The hard-core effects lead to packing effects that overcompensate the electrostatic correlations. For $a=0.4 r_{0}$, the density profiles are well captured by WDA0, which is always much closer to the MC data than to the PB result (not shown here). However, for $a=0.6 r_{0}$, the structure of packing becomes important at $\Gamma_{2 d}=2.0$. For this system, the WDA2 weight improves the result, showing that the deviation between the simulations and the constantweight WDA0 is not due to the electrostatic correlations but rather to hard-core effects. Beyond this point a more sophisticated functional should be used to capture the local packing.

In principle, the addition of salt can lead to new correlations due to ion-ion correlations and screening. For high electrostatic salt couplings, $\Gamma_{s}=\ell / d$, where $d$ is the distance of closest approach of ion and coion, ion clusters can also

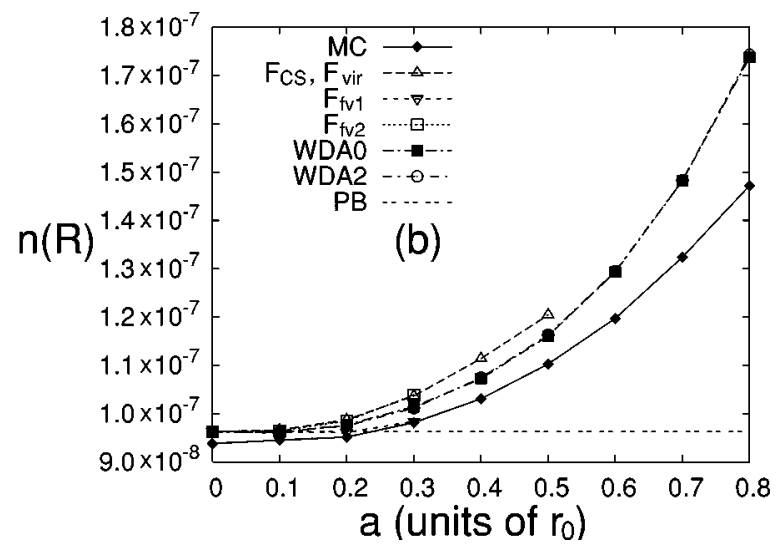

FIG. 4. Contact density (a) $n\left(r_{0}\right)$ and boundary density (b) $n(R)$ predicted for systems with $\Gamma_{2 d}=0.5$ by different methods as a function of ionic diameter (the number density is measured in units of $r_{0}^{-3}$ ). All theories at $a=0$ are identical to PB theory, which slightly underestimates the contact density due to ignoring electrostatic correlations. This effect reduces as the ion size increases. The legend is organized similar to that of Fig. 2 with one more line WDA2 corresponding to the weight by Tarazona given by Eqs. (19)-(21). There is a slight positive difference in $n(R)$ predicted by WDA2 and WDA0 not seen clearly in (b) at this scale. 

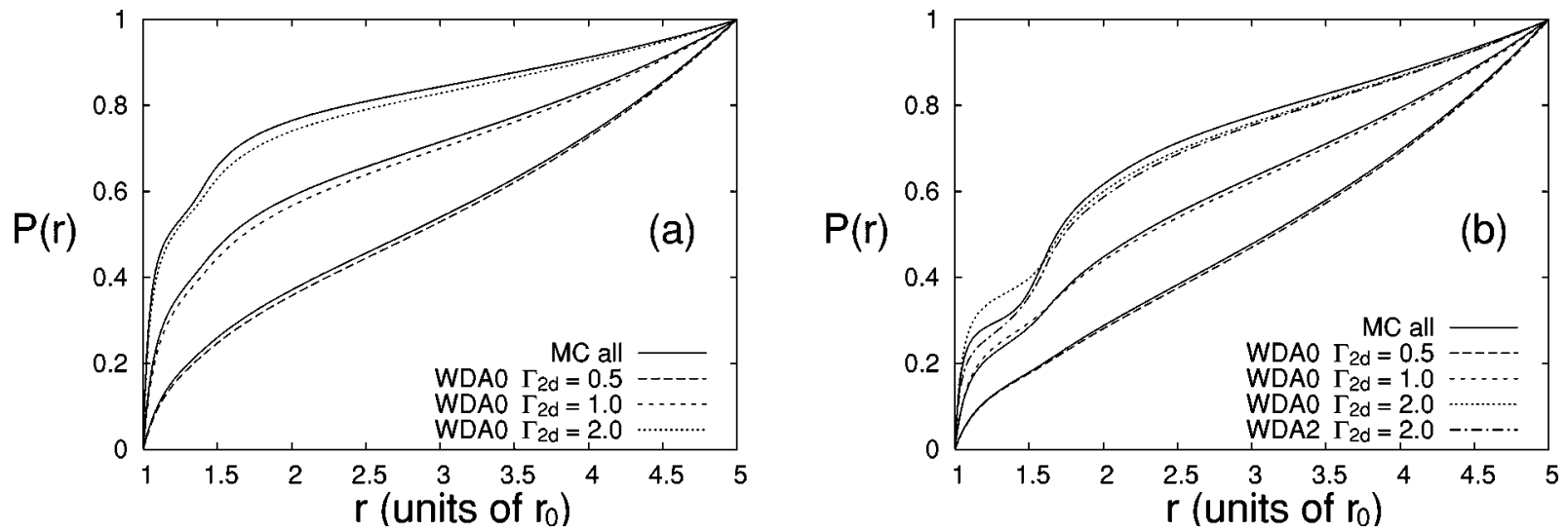

FIG. 5. Integrated fraction of counterions obtained using Monte Carlo (MC) and one constant-weighted-density approach (WDA0) for plasma parameter $\Gamma_{2 d}=0.5,1.0,2.0$ and for ionic diameters (a) $a=0.4 r_{0}$ and (b) $a=0.6 r_{0}$. In (b), the WDA2 curve is also shown for the case of $\Gamma_{2 d}=2.0$.

appear, which change the ion distribution considerably $[56,57]$. However, this effect can be overcome by the hard core if the ions are large enough, rendering $\Gamma_{s} \lesssim 1$. Below we consider the systems from Table I with added salt. Two cases of $N_{s}=10$ (10\% of salt) and $N_{s}=N(100 \%$ of salt) are studied to represent moderate and high amounts of salt. Since addition of salt ions into the cell would increase the packing fraction, here we prefer to keep it constant, adjusting accordingly the cell radius $R$. This is partially justified by the fact that the ion distribution close to the colloid weakly depends on the cell size for our system parameters. Figure 6 shows both the positive and negative charge density profiles obtained using simulations, WDA0, and PB theory for the system with $\Gamma_{2 d}=1.0$ and $a=0.4 r_{0}$ with $10 \%$ of salt. Due to the screening, the effect of electrostatic correlations is less profound than in the zero-salt case. The agreement between the simulations and WDA is therefore improved at higher salt concentrations and lower plasma parameter. The integrated charge profiles for several systems, employing our highest plasma parameter $\Gamma_{2 d}=2.0$, are shown in Fig. 7. The WDA captures the same trend in the charge distribution as provided by the MC data. The presence of salt does not change the

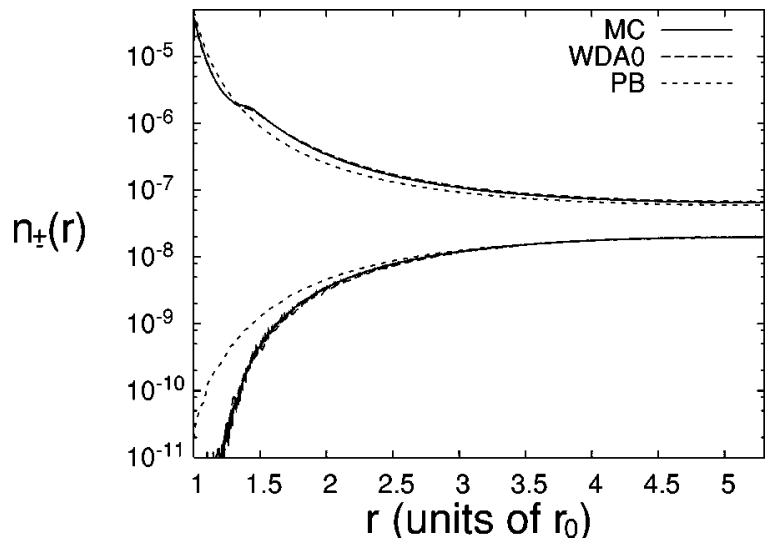

FIG. 6. Density profiles of positive and negative ions obtained using MC simulations, WDA0, and PB theory for the system with $\Gamma_{2 d}=1.0, a=0.4 r_{0}$, and $10 \%$ of salt. The number density is measured in units of $r_{0}^{-3}$. layering as is observed for this ionic radius when salt is not present. One should be aware that things will be more complicated if one considers asymmetric salt (in valence and size) or large ion sizes, since then more complicated effects like overcharging can occur [41,56-58].

\section{CONCLUSION}

In this paper we studied the effects of adding various local and nonlocal free-energy functionals to the PB free-energy functional to include the effects of an ionic hard core. We started from calculating a layer volume fraction $\phi_{s}$ using the PB approximation, which gives a criterion for recognizing when size effects become relevant and the simple PB approach has to be modified. We tested this criterion for a system consisting of a charged spherical colloid and its counterions confined to a spherical cell, and studied a number of parameter combinations where the PB approximation fails.

For including size correlations, four local and two nonlocal density-functional approximations were employed. The local theories were always found to overestimate the hardcore effects, creating an exclusion region close to the colloid for large ionic radii. Beyond a certain ionic radius, all the considered LDA's diverge and produce meaningless results. The failure of the LDA is also captured by the increasing divergence between the LDA and MC contact densities, which is seen when the ionic radius is increased, and the absence of any layering effect in the LDA. Due to this observation, we note that the inclusion of the LDA correction into PB actually worsens the agreement of PB with simulation results. In principle, a number of weighted density functionals $[22,34,51]$ or other nonlocal strategies [59] could be used in order to study this problem. We demonstrated that a simple weighted-density approach for the excluded-volume interaction was able to capture the main features of the ionic density profile. The introductions of a more sophisticated weighted-density approximations such as the approach of Tarazona et al. [34] improves the agreement with the simulation, but it does not bring any new physics to the problem. If some salt is included, under certain parameters the main effect is the increase of screening of the electrostatic corre- 

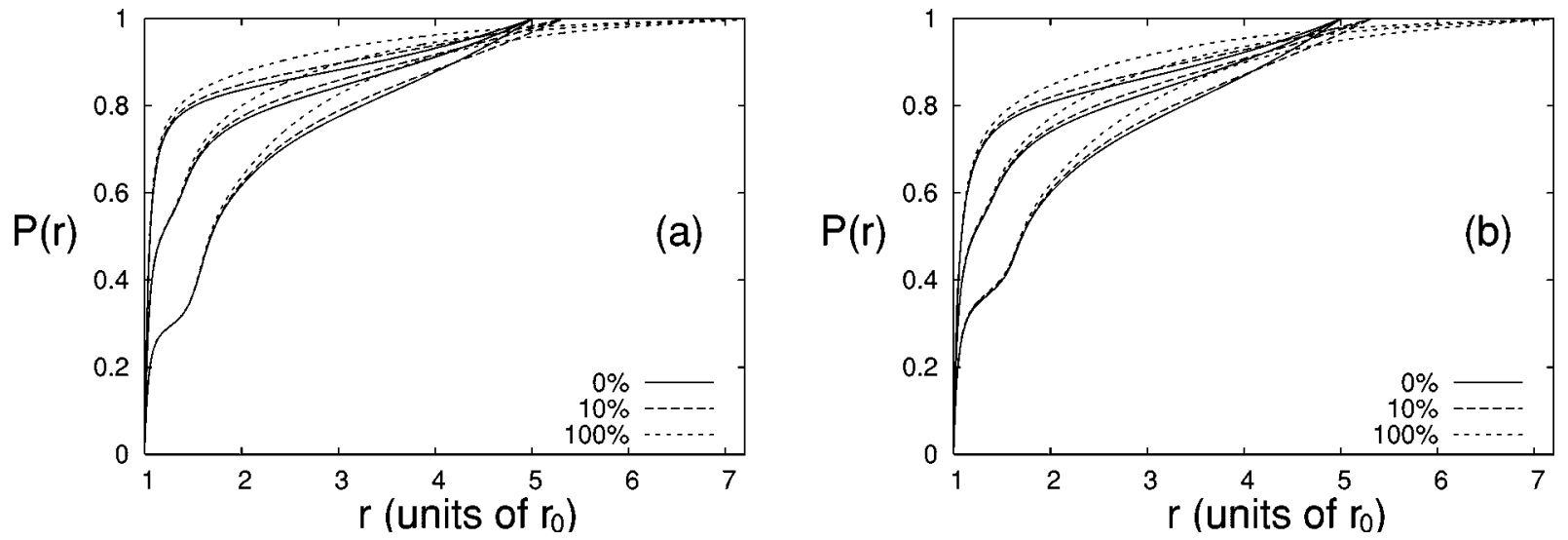

FIG. 7. Integrated fraction of counterions obtained using (a) Monte Carlo (MC) and (b) one constant-weighted-density approach (WDA0) for different ion sizes at plasma parameter $\Gamma_{2 d}=2.0$ and different amount of salt $\left(N_{s}=0,10 \%\right.$, and $100 \%$ of $\left.N\right)$. The sizes vary from top to bottom as $a=0.1 r_{0}, a=0.4 r_{0}$, and $a=0.6 r_{0}$.

lations. Therefore, the system can be adequately described by the PB approach supplemented with an excluded-volume WDA. More complicated effects are expected to appear at sufficiently high plasma parameters and higher salt concentrations. To treat those within density-functional theory, a combination of hard-core and electrostatic correlations along the lines of Refs. [22,51] will probably be required.

\section{ACKNOWLEDGMENTS}

This work has been supported by the Brazilian agencies, CNPq (Conselho Nacional de Desenvolvimento Científico e Tecnológico), and Capes through Grant No. 210-05 and the German Science Foundation (DFG) through Grant Nos. SFB 625, TR6 and Ho-1108/11-1, and PROBRAL Contract No. PO-D-04-40434. We would like to acknowledge M. Deserno for helpful discussions, and M.C.B. would also like to thank Professor K. Kremer for hospitality during her stay in Mainz.

\section{APPENDIX}

The canonical partition function $\mathcal{Z}$ of the colloid surrounded by its counterion in a cell model is given by

$$
\mathcal{Z}=\int \prod_{i=1}^{N+N_{s}} \prod_{j=1}^{N_{s}} \frac{d^{3} p_{i} d^{3} r_{i} d^{3} p_{j} d^{3} r_{j}}{h^{3\left(N+N_{s}\right)} h^{3 N_{s}}\left(N+N_{s}\right) ! N_{s} !} e^{-\beta \mathcal{H}}
$$

where $N=Z / v$ is the total number of counterions and $2 N_{s}$ is the total number of positive and negative ions of salt. The Hamiltonian $\mathcal{H}=\mathcal{T}+\mathcal{V}$ splits into kinetic and potential degrees of freedom. In the classical description employed here the kinetic part $\mathcal{T}$ will contribute the usual factor $\lambda^{-3 N-6 N_{s}}$ to the partition function, where $\lambda$ is the thermal de Broglie wavelength. The potential energy can be expressed as

$$
\begin{aligned}
\mathcal{V}= & -N \sum_{i}^{N+N_{s}} \frac{\ell}{\left|\mathbf{r}_{i}\right|}+N \sum_{i}^{N_{s}} \frac{\ell}{\left|\mathbf{r}_{i}\right|}+\frac{1}{2} \sum_{i \neq j}^{N_{s}, N_{s}} \frac{\ell}{\left|\mathbf{r}_{i}-\mathbf{r}_{j}\right|} \\
& +\frac{1}{2} \sum_{i \neq j}^{N_{s}+N, N_{s}+N} \frac{\ell}{\left|\mathbf{r}_{i}-\mathbf{r}_{j}\right|}-\frac{1}{2} \sum_{i \neq j}^{N_{s}, N_{s}+N}\left[\frac{\ell}{\left|\mathbf{r}_{i}-\mathbf{r}_{j}\right|}\right. \\
& \left.+g\left(\left|\mathbf{r}_{i}-\mathbf{r}_{j}\right| / a\right)\right],
\end{aligned}
$$

where the first two terms are related to the electrostatic interactions and the last is responsible for the hard-core repulsion. The specific form of this term is not relevant here. After rescaling all length by $\ell-$ i.e., introducing $\hat{\mathbf{r}}:=\mathbf{r} / \ell$ - the total partition function can be rewritten as

$$
\begin{aligned}
\mathcal{Z}= & \frac{1}{\left(N+N_{s}\right) ! N_{s} !}\left(\frac{\ell}{\lambda}\right)^{3 N+6 N_{s}} \int_{\hat{r}_{0}}^{\hat{r}_{0} / \phi^{1 / 3}} \prod_{k}^{N+N_{s}} \prod_{l}^{N_{s}} \int d^{3} \hat{r}_{k} d^{3} \hat{r}_{l} \\
& \times \exp \left\{-N \sum_{i}^{N+N_{s}} \frac{1}{\left|\hat{\mathbf{r}}_{i}\right|}+N \sum_{i}^{N_{s}} \frac{1}{\left|\hat{\mathbf{r}}_{i}\right|}+\frac{1}{2} \sum_{i \neq j}^{N_{s}+N_{,}+N_{s}+N} \frac{1}{\left|\hat{\mathbf{r}}_{i}-\hat{\mathbf{r}}_{j}\right|}\right. \\
& \left.+\frac{1}{2} \sum_{i \neq j}^{N_{s}, N_{s}} \frac{1}{\left|\hat{\mathbf{r}}_{i}-\hat{\mathbf{r}}_{j}\right|}-\frac{1}{2} \sum_{i \neq j}^{N_{s}+N, N_{s}}\left[\frac{1}{\left|\hat{\mathbf{r}}_{i}-\hat{\mathbf{r}}_{j}\right|}+g\left(\left|\hat{\mathbf{r}}_{i}-\hat{\mathbf{r}}_{j}\right| / \hat{a}\right)\right]\right\},
\end{aligned}
$$

where $\hat{a}=a / \ell$.

In this form it becomes evident that appropriately scaled thermal observables like the integrated charge density (measured in units of $\ell^{-3}$ ) or the pressure (measured in units of $k_{B} T \ell^{-3}$ ) are invariant under system changes which keep the number of counterions, $N$, the number of salt particles, $N_{s}$, the rescaled colloid size $\hat{r}_{0}=r_{0} / \ell$, the rescaled ion radius $\hat{a}$, and the volume fraction $\phi$ constant.

Poisson-Boltzmann theory shows the same invariance property, as does the approximate density functional theory we are proposing in this paper. 
[1] J.-L. Barrat and J.-F. Joanny, Adv. Chem. Phys. 94, 1 (1996).

[2] Polyelectrolytes: Science and Technology, edited by M. Hara (Marcel Dekker, New York, 1993).

[3] Electrostatic Effects in Soft Matter and Biophysics, edited by C. Holm, P. Kékicheff, and R. Podgornik, Vol. 46 of NATO Science Series II: Mathematics, Physics and Chemistry (Kluwer Academic, Dordrecht, 2001).

[4] A. P. Gast and W. B. Russel, Phys. Today 51, 24 (1998).

[5] D. Frenkel, Science 296, 65 (2002).

[6] O. D. Velev and E. W. Kaler, Adv. Mater. (Weinheim, Ger.) 12, 531 (2000).

[7] R. J. Ellis and A. P. Minton, Nature (London) 425, 27 (2003).

[8] A. P. Minton, Biophys. Chem. 57, 1 (1995).

[9] E. González-Tovar and M. Lozada-Cassou, J. Chem. Phys. 83, 361 (1985).

[10] M. Lozada-Cassou and E. Gonzáles-Tovar, J. Colloid Interface Sci. 239, 285 (2001).

[11] M. Tanaka and A. Y. Grosberg, Eur. Phys. J. E 7, 371 (2002).

[12] V. Lobaskin, B. Dünweg, and C. Holm, J. Phys.: Condens. Matter 16, S4063 (2004).

[13] K.-L. Yang, S. Yiacoumi, and C. Tsouris, J. Chem. Phys. 117, 8499 (2002).

[14] G. L. Gouy, J. Phys. (Paris) 9, 457 (1910).

[15] D. L. Chapman, Philos. Mag. 25, 475 (1913).

[16] R. M. Fuoss, A. Katchalsky, and S. Lifson, Proc. Natl. Acad. Sci. U.S.A. 37, 579 (1951).

[17] T. Alfrey, P. W. Berg, and H. J. Morawetz, J. Polym. Sci. 7, 543 (1951).

[18] A. Y. Grosberg, T. T. Nguyen, and B. I. Shklovskii, Rev. Mod. Phys. 74, 329 (2002).

[19] Y. Levin, Rep. Prog. Phys. 65, 1577 (2002).

[20] H. Löwen, J.-P. Hansen, and P. A. Madden, J. Chem. Phys. 98, 3275 (1993).

[21] C. N. Patra and S. K. Ghosh, Phys. Rev. E 47, 4088 (1993).

[22] W. N. D. Gillespie and R. S. Eisenberg, Phys. Rev. E 68, 031503 (2003).

[23] J. W. Yan-Xin Yu and G.-H. Gao, J. Chem. Phys. 120, 7223 (2004).

[24] Z. Li and J. Wu, Phys. Rev. E 70, 031109 (2004).

[25] M. C. Barbosa, M. Deserno, and C. Holm, Europhys. Lett. 52, 80 (2000).

[26] M. C. Barbosa, M. Deserno, C. Holm, and R. Messina, Phys. Rev. E 69, 051401 (2004).

[27] E. E. Salpeter, Ann. Phys. (N.Y.) 5, 183 (1958).

[28] R. Abe, Prog. Theor. Phys. 22, 213 (1959).

[29] M. Baus and J.-P. Hansen, Phys. Rep. 59, 1 (1980).

[30] R. D. Groot, J. Chem. Phys. 95, 9191 (1991).

[31] R. Penfold, S. Nordholm, B. Jönsson, and C. E. Woodward, J. Chem. Phys. 92, 1915 (1990).
[32] I. Borukhov, D. Andelman, and H. Orland, Phys. Rev. Lett. 79, 435 (1997).

[33] I. Borukhov, J. Polym. Sci., Part B: Polym. Phys. 42, 3598 (2004).

[34] P. Tarazona, R. Evans, and U. Marconi, Mol. Phys. 54, 1357 (1985).

[35] P. Tarazona, Mol. Phys. 52, 81 (1984).

[36] P. Tarazona, U. M. B. Marconi, and R. Evans, Mol. Phys. 60, 573 (1987).

[37] A. G. Moreira and R. R. Netz, Eur. Phys. J. E 8, 33 (2002).

[38] M. Deserno, A. Arnold, and C. Holm, Macromolecules 36, 249 (2003).

[39] I. Rouzina and V. Bloomfield, J. Phys. Chem. 100, 9977 (1996).

[40] B. Jönsson and H. Wennerström, in Electrostatic Effects in Soft Matter and Biophysics, edited by C. Holm, P. Kékicheff, and R. Podgornik, Vol. 46 of NATO Science Series II: Mathematics, Physics and Chemistry (Kluwer Academic, Dordrecht, 2001).

[41] R. Messina, E. G. Tovar, M. Lozada-Cassou, and C. Holm, Europhys. Lett. 60, 383 (2002).

[42] H. Wennerström, B. Jönsson, and P. Linse, J. Chem. Phys. 76, 4665 (1982).

[43] N. Grønbech-Jensen, R. J. Mashl, R. F. Bruinsma, and W. M. Gelbart, Phys. Rev. Lett. 78, 2477 (1997).

[44] N. F. Carnahan and K. E. Starling, J. Chem. Phys. 51, 535 (1969).

[45] D. A. McQuarrie, Statistical Mechanics, Harper's Chemistry Series (Harper Collins, New York, 1976).

[46] J. P. Hansen and I. McDonald, Theory of Simple Liquids (Academic, London, 1990).

[47] P. Tarazona, Phys. Rev. A 31, 2672 (1985).

[48] Y. Rosenfeld, Phys. Rev. Lett. 63, 980 (1989).

[49] Y. Rosenfeld, J. Chem. Phys. 98, 8126 (1993).

[50] S. Nordholm, M. Johnson, and B. C. Freasier, Aust. J. Chem. 33, 2139 (1980).

[51] Y.-X. Yu and J. Wu, J. Chem. Phys. 117, 10156 (2002).

[52] M. S. Wertheim, Phys. Rev. Lett. 10, 321 (1963).

[53] J. K. Percus and G. J. Yevick, Phys. Rev. 110, 1 (1958).

[54] M. Deserno, Physica A 278, 405 (2000).

[55] G. Téllez and E. Trizac, J. Chem. Phys. 118, 3362 (2003).

[56] E. Gonzales-Tovar, M. Lozada-Cassou, and D. Henderson, J. Chem. Phys. 83, 361 (1985).

[57] M. Deserno, F. Jiménez-Ángeles, C. Holm, and M. LozadaCassou, J. Phys. Chem. B 105, 10983 (2001).

[58] H. Greberg and R. Kjellander, J. Chem. Phys. 108, 2940 (1998).

[59] Y. Burak and D. Andelman, Phys. Rev. E 62, 5296 (2000). 